\title{
ORIGINAL ARTICLE \\ Nuevas citas e incidencias en parques eólicos de Nóctulo pequeño (Nyctalus leisleri, Khul 1817), Nóctulo mediano (Nyctalus noctula, Schreber 1774) y Nóctulo grande (Nyctalus lasiopterus, Schreber 1780) en la provincia de Soria (España)
}

\author{
Alberto De la Cruz Sánchez ${ }^{1}$, Manuel Meijide Fuentes ${ }^{2, *}$, Federico Meijide Fuentes ${ }^{3}$
}

\author{
${ }^{1}$ dubhe00@gmail.com \\ ${ }^{2}$ C/ Felicidad, 85, 42190 Urb. Las \\ Camaretas, Golmayo, Soria, España. \\ ${ }^{3}$ C/ Jueves La Saca, 1, 10 izq. 42002 \\ Soria, España \\ *Corresponding author: \\ manuelmeijide@hotmail.com \\ DOI: https://doi.org/10.14709/ \\ BarbJ.13.1.2020.03 \\ Palabras clave: Castilla y León, \\ distribución, murciélagos forestales, \\ nóctulos, parques eólicos, Soria.
}

Keywords: Castilla y León, distribution, forest bats, noctules, Soria, wind farms.

received: April, 26th 2019

accepted: March, 23rd 2020

\begin{abstract}
RESUMEN
Los quirópteros forestales han sido, en general, poco estudiados hasta la aparición de los detectores de ultrasonidos y los programas de reconocimiento e identificación de vocalizaciones, lo que ha facilitado su detectabilidad y estudio. Además, a nivel peninsular los datos y registros de estos murciélagos son relativamente escasos. Con esta nota ampliamos el rango de distribución de los nóctulos en la península ibérica y la información sobre incidencias en parques eólicos. La presencia en la provincia de Soria de nóctulo pequeño (Nyctalus leisleri) y nóctulo mediano (Nyctalus noctula) es por el momento anecdótica, mientras que para el nóctulo grande (Nyctalus lasiopterus) es algo más frecuente. Recientemente se ha descrito la presencia de una agrupación de nóctulo mediano en Almazán (Soria) con avistamientos desde 2016, así como de nóctulo pequeño y grande en la zona conocida como Pinar Grande al noroeste de Soria. En este trabajo aportamos nuevas citas procedentes de estudios de siniestralidad en instalaciones eólicas de la provincia obtenidas de la base de datos de la Junta de Castilla y León, donde se constata la presencia de estas especies entre 2005 y 2019, así como otras citas recopiladas en los últimos años a través de muestreo mediante redes y detectores de ultrasonidos. Los ejemplares de nóctulo mediano encontrados durante los estudios en parques eólicos en el sur de la provincia que pudieron ser sexados ( $26 \%$ del total de los siniestros) eran todos machos probablemente machos en fase de apareamiento provenientes del parque de La Arboleda de Almazán. En el caso del nóctulo grande, las citas resultaron dispersas por la provincia, pero recurrentes a lo largo de los últimos años. En cambio, las citas de nóctulo pequeño fueron especialmente escasas en parques eólicos.
\end{abstract}

\begin{abstract}
Forest chiropterans have in general, been little studied until the appearance of ultrasound detectors and vocalization recognition and identification programs, which has facilitated their detectability and study. In addition, at the peninsular level, the data and records of these bats are relatively scarce. With this note we expand the range of distribution of the Noctules bats in the Iberian Peninsula and the information on incidents in wind turbines. The presence in the province of Soria of Nyctalus leisleri and Nyctalus noctula is at the moment anecdotal, while for the Nyctalus lasiopterus it is somewhat more frequent. Recently it has been described the presence of an agrupation of Nyctalus noctula in Almazán (Soria), has been described with sightings since 2016, as well as a Nyctalus leisleri and Nyctalus lasiopterus in the area known as Pinar Grande to the northwest of Soria. In this work we provide new citations from accident studies in wind farms in the province obtained from the database of the Junta de Castilla y León, where the presence of these species is verified between 2005 and 2019, as well as other citations collected in the last years through sampling using networks and ultrasound detectors. The specimens of Nyctalus noctula found during the studies in wind farms in the south of the province that could be sexed ( $26 \%$ of the total losses) were all males, probably males in the mating phase from the La Arboleda de Almazán park. In the case of the Nyctalus lasiopterus, the citations were scattered throughout the province, but recurring in recent years. In contrast, small nodule appointments were especially rare in wind turbines.
\end{abstract}




\section{INTRODUCCIÓN}

Debido a la dificultad para localizar y capturar murciélagos forestales, existe poca información sobre la distribución y conservación en su medio. El ambiente forestal resulta necesario para la vida de muchas especies de quirópteros, ya sea para cazar, hibernar, reproducirse o como refugio temporal. Además la buena salud de los recursos forestales, garantiza la diversidad de especies que lo ocupan, indicando que si la abundancia de especies es alta, la calidad estructural del bosque es buena. Se sabe que estas poblaciones se ven afectadas por diversas causas como, por ejemplo, la deforestación o la tala de árboles viejos con huecos que ofrecen mayor cantidad de refugios. Evidentemente los incendios y los pesticidas para el control de plagas también originan graves prejuicios sobre los quirópteros. Además recientemente, la proliferación de parques eólicos en algunas zonas de migración o en zonas cercanas a colonias de cría puede mermar significativamente algunas poblaciones (Brinkmann et al. 2011, Guixé \& Camprodon 2018). Por ello, la información de los hábitats y de la localización donde los aerogeneradores pueden ocasionar impacto sobre los quirópteros debería emplearse en la toma de decisiones de los proyectos eólicos (Rodrigues et al. 2015).

Hay poca bibliografía acerca de qué tipo de actuaciones habría que realizar en los parques eólicos para minimizar la mortalidad, aunque algunas medidas han resultado eficaces. Experimentos llevados a cabo para modificar el arranque de los aerogeneradores únicamente a velocidades de viento superiores a los 5-6 m/s en los períodos de mayor mortalidad (meses de julio a octubre) (Baerwald et al. 2009, Arnett et al. 2011) han demostrado que ésta es, por el momento, la medida más eficaz para reducirla. Otras medidas para tratar de disminuir la actividad de los murciélagos en las proximidades de los aerogeneradores como la emisión de ruido blanco ultrasónico (Horn et al. 2008, Szewczak \& Arnett 2008, Johnson et al. 2012) o la aplicación de radiación electromagnética de alta frecuencia (Nicholls \& Racey 2007, 2009) parece que podrían tener efectos disuasorios, pero por el momento los resultados no son concluyentes.

Por otra parte, los parques eólicos suponen actualmente una herramienta de información sobre la distribución de diferentes especies de quirópteros a través de los animales siniestrados, datos que pueden ser muy relevantes en algunas zonas poco prospectadas por la dificultad de observar, escuchar o capturar a algunas especies, como ha sido el caso de los murciélagos analizados en este artículo.

Nyctalus lasiopterus y Nyctalus leisleri eran conocidos en la provincia de Soria desde 1995 (Meijide et al. 1996, Fernández-Gutiérrez 2002), reconociéndose varias citas repartidas en el cuadrante noroccidental, en la zona conocida como Pinar Grande y el parque Natural del Cañón del Río Lobos. Sin embargo, la primera cita publicada de Nyctalus noctula en esta provincia de España es mucho más reciente y corresponde a una colonia de celo en la localidad de Almazán (Flechoso et al. 2018).

En esta nota presentamos la actualización de las distribuciones de Nyctalus lasiopterus, Nyctalus leisleri y Nyctalus noctula recopilada a través de muestreos realizados para la revisión del Atlas de los mamíferos de la provincia de Soria (Meijide et al. 1996) y de la base de datos de los planes de seguimiento de parques eólicos de la Junta de Castilla y León.

\section{MATERIALES Y MÉTODOS}

Para la realización del presente trabajo combinamos diferentes metodologías. Por un lado, consultamos los datos históricos, obtenidos de la base de datos de los planes de seguimiento de todos los parques eólicos de la provincia, a través de la Delegación Territorial de Medio Ambiente de Soria, perteneciente a la Consejería de Fomento y Medio Ambiente de la Junta Castilla y León. Los datos presentes en dicha base contenían generalmente las fechas de detección de los ejemplares para cada instalación eólica, así como el término municipal donde se encontraron, sexándose cuando el estado de conservación del ejemplar lo permitía. En las instalaciones eólicas que se han revisado, la frecuencia de búsqueda fue semanal durante los meses de marzo, abril, mayo, agosto, septiembre y octubre, y quincenal el resto del año. La búsqueda de cadáveres se efectuó mediante espirales de búsqueda en el entorno de los aerogeneradores con un promedio de 20 minutos por máquina y un área de prospección de al menos un $10 \%$ del diámetro del rotor. Los ejemplares siniestrados los identificamos in situ en base a la clave de identificación de Dietz \& Von Helversen (2005).

Por otro lado, complementamos la base de datos del Atlas de mamíferos existente con datos personales de los autores obtenidos a lo largo de los últimos 20 años, en los que figuran varios registros de quirópteros del género Nyctalus. Estos datos proceden de diferentes muestreos con red japonesa y, en los últimos años, con apoyo de detectores de ultrasonidos EM3 y Echo Meter Touch 2 Pro (Wildlife Acoustics, USA) y el software Batsound 5 (Pettersson Elektronics, Sweden) y Kaleidoscope 5.1.1. (Wildlife Acoustics, USA). Este Atlas se encuentra en revisión y tiene como número de expediente EP/SO/245/2018, concedido por el Servicio Territorial de Medio Ambiente de la Delegación Territorial de Soria.

\section{RESULTADOS Y DISCUSIÓN}

\section{Nyctalus leisleri}

En la provincia de Soria detectamos Nyctalus leisleri mediante muestreos específicos en relativamente pocas zonas (Fig. 1, Tabla 1). La especie ocupa los pisos de vegetación Supramediterráneo húmedo-subhúmedo y Supramediterráneo (Rivas-Martínez 1982). Se detectó en tres parques eólicos del noreste de la provincia, muy cercanos entre sí, y en un cuarto parque en el sur (Fig. 2).

El nóctulo pequeño (Nyctalus leisleri, Kuhl 1817), en la Península Ibérica tiene una distribución amplia pero discontinua. Parece más extendido en los sectores forestales de montaña o de tendencia eurosiberiana, donde llega a ser localmente abundante, ligado a la presencia de caducifolios, aunque es frecuente encontrarlo en pinares y en Andalucía, en bosques mediterráneos. La disponibilidad de buenas cavidades en árboles es un factor influyente en la selección del hábitat a pequeña escala (Agirre-Mendi 2007). 
En la Península Ibérica se encuentra presente todo el año, existiendo machos y hembras sedentarias en la mitad sur, y solo machos sedentarios en la mitad norte. Además, hay aportes de ejemplares migrantes tanto en otoño como en primavera. El apareamiento tiene lugar entre septiembre y octubre (Camprodon \& Guixé 2007, 2008). En la Península Ibérica solo se han encontrado hembras reproductoras en diferentes puntos de la mitad sur (Ibáñez et al. 1992, 2009). En el norte, las hembras, y probablemente algunos machos, llegan del centro de Europa tras el verano para aparearse y pasar el invierno, volviendo en primavera a las áreas de cría (Alcalde et al. 2013).

Esta especie está catalogada en el Catálogo Español de Especies Amenazadas (BOE 2011) como "De interés especial" y en el Atlas y Libro Rojo de los Vertebrados de España (Palomo et al. 2007) como "Casi amenazado".

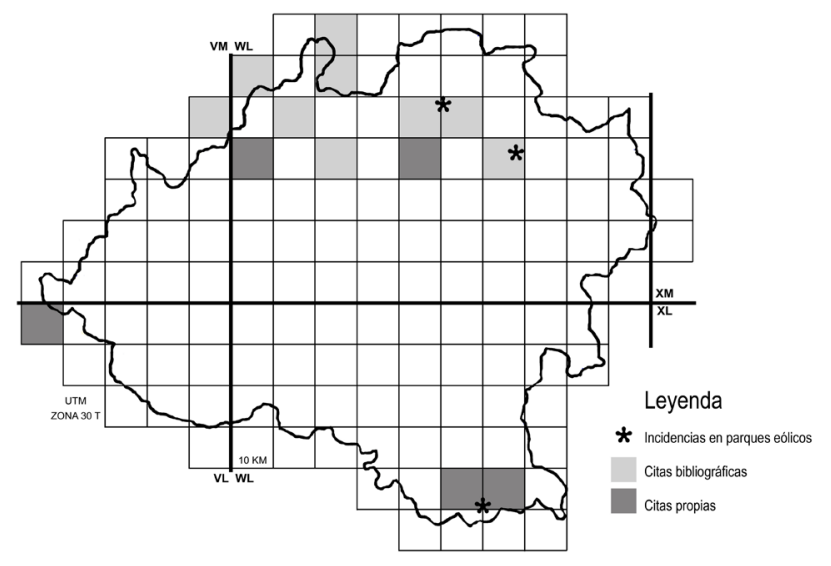

Fig. 1 - Mapa de distribución de Nyctalus leisleri en la provincia de Soria. Elaboración propia. Las cuadrículas en gris claro corresponden a citas bibliográficas (Meijide et al. 1996, FernándezGutiérrez 2002, Agirre-Mendi 2007, Junta de Castilla y León 2019), las cuadrículas en gris oscuro corresponden a muestreos específicos propios y los asteriscos corresponden a la base de datos de los planes de seguimiento de los parques eólicos (Junta de Castilla y León 2019).

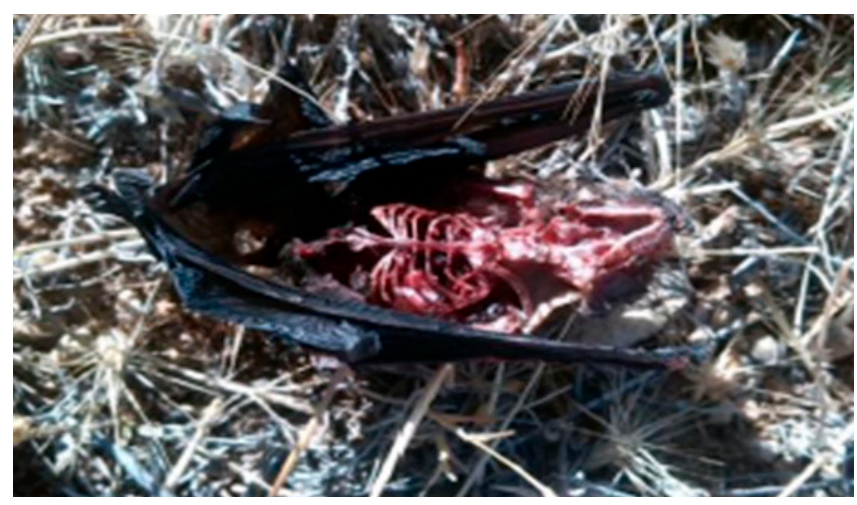

Fig. 2 - Detección de cadáver de Nyctalus leisleri, en Layna, 22/09/2016. Imagen procedente del archivo de TESTA SL.

\section{Nyctalus noctula}

En la provincia de Soria, se conocía la existencia de la especie desde 2015 por varios ejemplares hallados muertos en diversos parques eólicos de la zona centro-sur (Fig. 3). Además, en 2016 un ejemplar hallado muerto en las cercanías de Almazán fue depositado en la Consejería de Medio Ambiente de la Junta de Castilla y León. En la provincia soriana se encuentra en ambientes de vegetación Supramediterránea y Meso-supramediterránea (RivasMartínez 1982). Únicamente detectamos incidencias en tres parques eólicos (Fig. 4).

Como puede observarse, en septiembre de 2015 se registraron cuatro citas de machos en Morón de Almazán (Tabla 2), coincidiendo con la época de apareamiento de la especie. A unos 10 kilómetros de distancia se encuentra el parque de La Arboleda donde se describió la primera colonia de esta especie en Castilla y León (Flechoso et al. 2018). Las otras dos citas, en el municipio de Ventosa del Ducado y en Aldealpozo se encuentran a unos 40 kilómetros de Almazán.

El nóctulo mediano (Nyctalus noctula, Schreber 1774) es un murciélago forestal que acostumbra a refugiarse en huecos de árboles, producidos por podredumbres o pícidos, aunque también es posible hallarlo en cajas-refugio o en grietas de muros, edificios y puentes (Ruedi et al. 1998, Alcalde 1999). Presenta hábitos arborícolas y migradores (Sluiter \& Van Heerdt 1966, Rachwald 1992, Strelkov 2000).

En España se considera una especie rara, ya que, hasta el momento solo se han encontrado seis pequeñas agrupaciones: Aranjuez en Madrid (Ruedi et al. 1998), Pamplona y Sangüesa en Navarra (Alcalde 1999), Lleida (Camprodon \& Guixé 2013), Nuévalos en Zaragoza (Lorente et al. 2014) y Almazán en Soria (Flechoso et al. 2018). Las citas fiables en España son escasísimas y se reparten de forma muy dispersa (Alcalde 2002, 2007, Molleda \& Fombellida 2018). La colonia de Aranjuez es la única reproductora, existiendo en el resto exclusivamente machos sedentarios durante el verano. Durante la época de apareamiento (septiembrenoviembre) se pueden observar numerosas hembras, que llegan progresivamente desde finales de agosto (Alcalde 1999). Aunque se desconoce el origen de estas hembras, se supone que provienen de la mitad septentrional del continente, puesto que allí se concentra la mayoría de las colonias reproductoras y se han registrado movimientos migratorios de esta especie entre Holanda y el suroeste de Francia (Sluiter \& Van Heerdt 1966).

Esta especie está catalogada en el Catálogo Español de Especies Amenazadas (BOE 2011) como "vulnerable" y en el Atlas y Libro Rojo de los Vertebrados de España (Palomo et al.

Tabla 1 - Registro de citas de nóctulo pequeño (Nyctalus leisleri) procedentes de instalaciones eólicas en la provincia de Soria desde 2005 a 2016. Fuente: Delegación Territorial de Medio Ambiente en Soria. Consejería de Fomento y Medio Ambiente. Junta de Castilla y León (2019).

\begin{tabular}{lccc}
\hline Término municipal & Fecha & Edad & Sexo \\
\hline Las Aldehuelas & 2005 & Indeterminado & Indeterminado \\
Magaña & $10 / 09 / 2014$ & Adulto & Macho \\
Layna & $22 / 09 / 2016$ & Indeterminado & Indeterminado \\
\hline
\end{tabular}


Tabla 2 - Registro de citas de nóctulo mediano (Nyctalus noctula) siniestrados procedentes de instalaciones eólicas en la provincia de Soria desde 2015 a 2018. Fuente: Delegación Territorial de Medio Ambiente en Soria. Consejería de Fomento y Medio Ambiente. Junta de Castilla y León (2019).

\begin{tabular}{lccc}
\hline Término municipal & Fecha & Edad & Sexo \\
\hline Morón de Almazán & $04 / 09 / 2015$ & Indeterminado & Macho \\
Morón de Almazán & $04 / 09 / 2015$ & Indeterminado & Macho \\
Morón de Almazán & $09 / 09 / 2015$ & Indeterminado & Macho \\
Morón de Almazán & $09 / 09 / 2015$ & Indeterminado & Macho \\
Ventosa del Ducado & $10 / 09 / 2015$ & Indeterminado & Indeterminado \\
Morón de Almazán & $23 / 09 / 2015$ & Indeterminado & Indeterminado \\
Aldealpozo-Tajahuerce & $14 / 09 / 2018$ & Indeterminado & Indeterminado \\
\hline
\end{tabular}

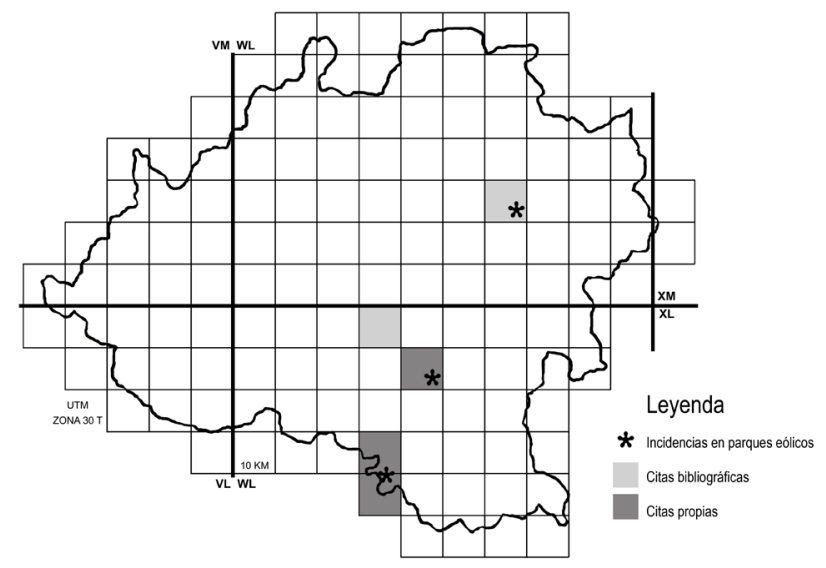

Fig. 3 - Mapa de distribución de Nyctalus noctula en la provincia de Soria. Elaboración propia. Las cuadrículas en gris claro corresponden a citas bibliográficas (Flechoso et al. 2018, Junta de Castilla y León 2019), las cuadrículas en gris oscuro corresponden a muestreos específicos propios y los asteriscos corresponden a la base de datos de los planes de seguimiento de los parques eólicos (Junta de Castilla y León 2019).

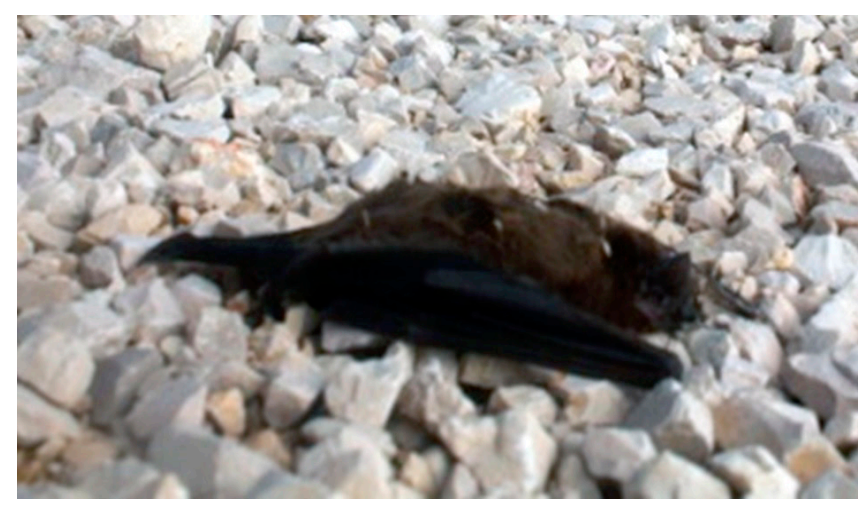

Fig. 4 - Detección de cadáver de Nyctalus noctula, en Morón de Almazán, 04/09/2015. Imagen procedente del archivo de TESTA SL.

2007) como "vulnerable" (VU B1 ab(iii); D1). Su catalogación en el Libro Rojo se explica por la pérdida comprobada de numerosos árboles-refugio debido a la tala de ejemplares viejos y con oquedades. Los parques eólicos son también una amenaza para estas poblaciones si se disponen a lo largo de sus rutas migratorias o en las cercanías de colonias. Se han recogido ejemplares muertos por interacción con los aerogeneradores en España (Alcalde \& Sáenz 2004, Guixé \& Camprodon 2018), Alemania y Suecia (Ahlén \& Gerell 1989).

\section{Nyctalus lasiopterus}

Basándose en los muestreos con red y detector realizados, así como por el registro de incidencias en parques eólicos de esta especie (Fig. 6), parece ser que la presencia de este Nyctalus es más frecuente que $N$. leisleri y $N$. noctula (Fig. 5). Ocupa los pisos de vegetación Crioromediterráneo, Supramediterráneo húmedo subhúmedo, Supramediterráneo semihúmedo, Supramediterráneo y Meso-supramediterráneo (RivasMartínez 1982).

Detectamos el nóctulo grande en puntos dispersos de la provincia de Soria, de forma continuada durante los últimos años, con presencia tanto de ejemplares machos como hembras, siendo interesante la presencia de individuos jóvenes durante algunas observaciones de 2014 (Tabla 3).

El nóctulo grande (Nyctalus lasiopterus, Schreber 1780), mantiene una distribución poco conocida. En España es posiblemente discontinua, con núcleos poco conocidos y citas puntuales (Juste 2007). Se conocen colonias de cría en el sur peninsular, en el Sistema Central y Aragón, siendo las poblaciones norteñas migradoras (Guixé \& Camprodon 2018). Esta especie está básicamente asociada a bosques de caducifolios (generalmente Quercus sp. y Fagus sy/vatica) aunque se ha encontrado también en pinares maduros (Pinus sylvestris) y criando en viejos plátanos y palmeras de parques (Camprodon \& Guixé 2013). Presenta una gran capacidad de desplazamiento que le permite explotar áreas de caza muy distantes de los refugios. En Andalucía forma colonias de cría de hasta 80 hembras que pueden utilizar distintos refugios próximos y que se disgregan al independizarse los jóvenes (Santos et al. 2016). En el norte de la Península Ibérica y Grecia, los individuos capturados en verano son casi exclusivamente machos, este desequilibrio entre sexos se ha asociado a movimientos migratorios estacionales similares a los conocidos para otros nóctulos (Guixé \& Camprodon 2018). Las áreas de campeo del nóctulo grande probablemente coinciden con zonas migratorias de insectos y/o aves. La dieta a base de paseriformes migratorios es más importante que la de insectos durante el otoño, como se ha apuntado para las poblaciones ibéricas (Ibáñez et al. 2001, Popa-Lisseanu et al. 2007). La especie puede recorrer hasta $40 \mathrm{~km}$ diarios, como se ha reportado en Sevilla (PopaLisseanu et al. 2009). Se han citado distancias de caza de 26 km (Gebhard \& Bogdanowicz 2004). Pero en áreas con alta densidad de presas las zonas de alimentación se concentran a una distancia menor de $25 \mathrm{~km}$ (Kronwitter 1988). 
Tabla 3 - Registro de citas de siniestros de nóctulo grande (Nyctalus lasiopterus) procedentes de instalaciones eólicas en la provincia de Soria desde 2005 a 2019. Fuente: Delegación Territorial de Medio Ambiente en Soria. Consejería de Fomento y Medio Ambiente. Junta de Castilla y León (2019).

\begin{tabular}{|c|c|c|c|}
\hline Término municipal & Fecha & Edad & Sexo \\
\hline Castro & $12 / 09 / 2005$ & Indeterminado & Indeterminado \\
\hline Las Aldehuelas & 27/08/2005 & Indeterminado & Indeterminado \\
\hline Castro & $19 / 09 / 2007$ & Indeterminado & Indeterminado \\
\hline Morón de Almazán & $08 / 10 / 2007$ & Indeterminado & Indeterminado \\
\hline Bordecorex & $11 / 07 / 2008$ & Indeterminado & Indeterminado \\
\hline Oncala & $23 / 09 / 2008$ & Indeterminado & Indeterminado \\
\hline Radona & 2009 & Indeterminado & Indeterminado \\
\hline Morón de Almazán & $24 / 08 / 2010$ & Indeterminado & Indeterminado \\
\hline Morón de Almazán & $18 / 08 / 2011$ & Indeterminado & Indeterminado \\
\hline Ontalvilla de Almazán & $12 / 09 / 2013$ & Indeterminado & Indeterminado \\
\hline Suellacabras & $28 / 08 / 2014$ & Indeterminado & Indeterminado \\
\hline Los Campillos & $02 / 09 / 2014$ & Joven & Macho \\
\hline Los Campillos & 04/09/2014 & Indeterminado & Indeterminado \\
\hline Morón de Almazán & $10 / 09 / 2014$ & Joven & Macho \\
\hline Bordecorex norte & $16 / 09 / 2014$ & Indeterminado & Indeterminado \\
\hline Ontalvilla de Almazán & $29 / 10 / 2014$ & Joven & Indeterminado \\
\hline Morón de Almazán & 09/09/2015 & Indeterminado & Indeterminado \\
\hline Retortillo de Soria & 01/08/2016 & Indeterminado & Hembra \\
\hline Portelrubio & $05 / 10 / 2016$ & Indeterminado & Indeterminado \\
\hline Magaña & $25 / 08 / 2017$ & Indeterminado & Indeterminado \\
\hline Trévago & 2017 & Indeterminado & Indeterminado \\
\hline Magaña & $02 / 08 / 2017$ & Indeterminado & Indeterminado \\
\hline Radona & $31 / 08 / 2017$ & Indeterminado & Macho \\
\hline Radona & $14 / 09 / 2017$ & Indeterminado & Indeterminado \\
\hline Layna & $23 / 08 / 2018$ & Indeterminado & Indeterminado \\
\hline Retortillo de Soria & $27 / 08 / 2018$ & Indeterminado & Hembra \\
\hline Suellacabras & 18/09/2019 & Indeterminado & Indeterminado \\
\hline
\end{tabular}

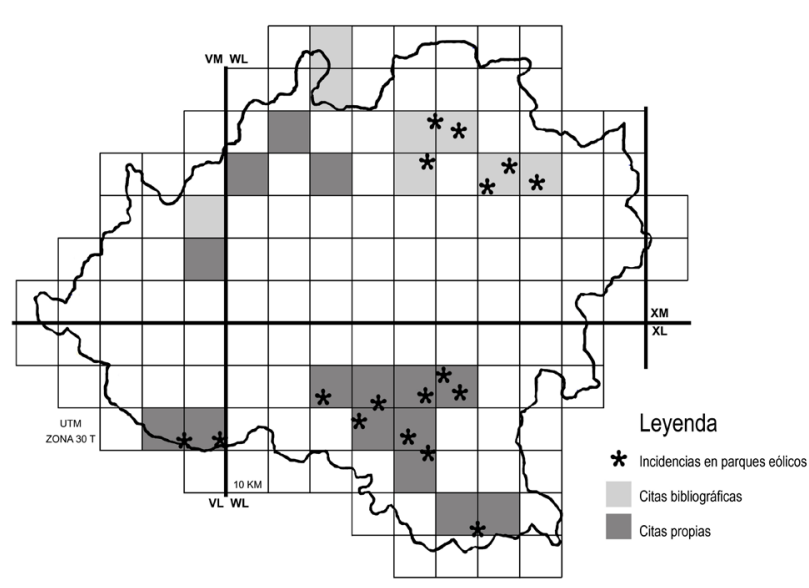

Fig. 5 - Mapa de distribución del Nyctalus lasiopterus en Soria. Elaboración propia. Las cuadrículas en gris claro corresponden a citas bibliográficas (Meijide et al. 1996, Fernández-Gutiérrez 2002, Junta de Castilla y León 2019), las cuadrículas en gris oscuro corresponden a muestreos específicos propios y los asteriscos corresponden a la base de datos de los planes de seguimiento de los parques eólicos (Junta de Castilla y León 2019).

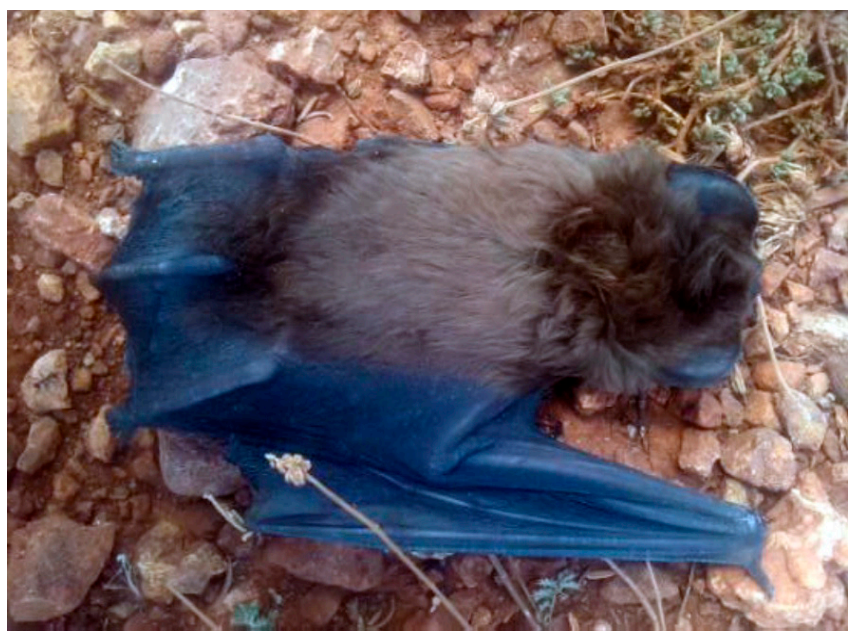

Fig. 6 - Detección de Nyctalus lasiopterus, en Retortillo de Soria, 01-08-2016. Imagen procedente del archivo de TESTA SL. 
Esta especie está catalogada en el Catálogo Español de Especies Amenazadas (Real Decreto 439/1990) como "vulnerable" y en el Atlas y Libro Rojo de los Vertebrados de España (Palomo et al. 2007) como "vulnerable".

\section{CONCLUSIONES}

Estas nuevas aportaciones amplían la distribución conocida de estas especies en la provincia de Soria. El conocimiento de cómo afectan las instalaciones eólicas a estos murciélagos en la provincia sugiere que en otros parques eólicos, menos investigados, se produzcan también estos siniestros. En estos parques han aparecido siniestradas otras especies de quirópteros como Pipistrellus pipistrellus, Pipistrellus pygmaeus, Pipistrellus kuhlii, Hypsugo savi, Verpertilio murinus, Tadarida teniotis, Miniopterus schreibersi y Myotis myotis.

Aunque aparentemente la frecuencia relativamente baja de incidencias no parece relevante, probablemente la presencia de carroñeros supone la pérdida de ejemplares muertos, siendo imprescindibles los ensayos de detectabilidad y permanencia de los cadáveres (Rodrigues et al. 2015). Además, en las poblaciones locales estas bajas pueden ser significativas en proporción al tamaño habitualmente reducido de sus colonias. En estos casos, es fundamental aplicar medidas preventivas para permitir la supervivencia de colonias como en el caso de la colonia de Nyctalus noctula de la Arboleda de Almazán que se encuentra a 10 kilómetros de distancia de varios parques eólicos.

\section{AGRADECIMIENTOS}

Hay que agradecer a la empresa TESTA S.L. por facilitar las fotografías y el conocimiento de las metodologías empleadas en la búsqueda original de los ejemplares, y, en especial, a los compañeros Lidia Díaz, Mónica Mencía, Ángel Rubio y David Merino, sin cuya colaboración se hubiese dificultado enormemente nuestro trabajo. A Carolina Fuentes y Lucía Jiménez por sus aportaciones. A la Asociación AMAR Soria por sus citas y conocimientos. Y a la Junta de Castilla y León, Servicio Territorial de Medio Ambiente de la Delegación Territorial de Soria, por su colaboración aportando las citas de las incidencias de quirópteros en parques eólicos. Finalmente, agradecemos a los revisores del artículo sus aportaciones al mismo.

\section{REFERENCIAS}

AGIRRE-MENDI, P. T. (2007). Nyctalus leisleri (Kuhl 1817). En: Atlas y Libro Rojo de los Mamíferos Terrestres de España. ed.: Dirección General para la Biodiversidad-SECEMSECEMU. Madrid, España, p.222-227.

AHLÉN, I. \& GERELL, R. (1989). Distribution and status of bats in Sweden. In: European Bat Research 1987. ed.: Charles University Press. Prague, Czech Republic , p.319-325.
ALCALDE, J. T. (1999). New ecological data on the noctule bat (Nyctalus noctula Schreber, 1774) (Chiroptera, Vespertilionidae) in two towns of Spain. Mammalia, 63(3): 273-280. https://doi.org/10.1515/mamm.1999.63.3.273

ALCALDE, J. T. (2002). Nyctalus noctula (Schreber, 1774). En: Atlas de los Mamíferos Terrestres de España. ed.: Dirección General para la Biodiversidad-SECEM-SECEMU. Madrid, España, p.198-201.

ALCALDE, J. T. \& SÁENZ, J. (2004). First data on bat mortality in wind farms of Navarre (Northern Iberian Peninsula). Le Rinolophe, 17: 1-5.

ALCALDE, J. T. (2007). Nyctalus noctula (Schreber, 1774). En: Atlas y Libro Rojo de los Mamíferos Terrestres de España. ed.: Dirección General para la Biodiversidad-SECEMSECEMU. Madrid, España, p. 228-232.

ALCALDE, J. T., IBAÑEZ, C., ANTÓN, I. \& NYSSEN, P. (2013). First case of migration of a Leisler's bat (Nyctalus leisleri) between Spain and Belgium. Le Rhinolophe, 19: 87-88.

ARNETT, E.B., HUSO, M.M., SCHIRMACHER, M.R., HAYES, J.P. (2011). Altering turbine speed reduces bat mortality at wind-energy facilities. Frontiers in Ecology and the Environment, 9(4):209-214. https://doi. org/10.1890/100103

BAERWALD, E. F., EDWORTHY, J., HOLDER, M. \& BARCLAY, R. M. R. (2009). A large-scale mitigation experiment to reduce bat fatalities at wind energy facilities. The Journal of Wildlife Management , 73(7): 1077-1081. https://doi. org/10.2193/2008-233

BOLETÍN OFICIAL DEL ESTADO (BOE) (2011). Real Decreto 139/2011, de 4 de febrero, para el desarrollo del Listado de Especies Silvestres en Régimen de Protección Especial y del Catálogo Español de Especies Amenazadas.

BRINKMANN, R., BEHR, O., NIERMANN, I. \& REICH, M. (2011). Entwicklung von methoden zur untersuchung und reduktion des kollisionsrisikos von fledermäusen an Onshore-Windenergieanlagen. ed.: Cuvillier Verlag. Göttingen, Germany, 470 pp.

CAMPRODON, J. \& GUIXÉ, D. (2013). Estado poblacional, selección de refugios y ecología espacial de las poblaciones de nóctulo grande (Nyctalus lasiopterus) y nóctulo mediano (Nyctalus noctula) en Cataluña. Journal of Bat Research \& Conservation, 6(1): 53-61. https://doi. org/10.14709/BarbJ.6.1.2013.07

DIETZ, C., \& VON HELVERSEN, O. (2005). Claves de identificación ilustradas de los murciélagos de Europa. Publicación electrónica.

FERNÁNDEZ-GUTIÉRREZ, J. (2002). Los Murciélagos en Castilla y León: atlas de distribución y tamaño de las poblaciones. ed.: Junta de Castilla y León, Consejería de Medio Ambiente. Valladolid, España, 360 pp. 
FLECHOSO, M., FERNÁNDEZ, D. \& ALCALDE, J. T. (2018). Primera colonia de nóctulos medianos (Nyctalus noctula Schreber, 1774) en Castilla y León (Norte de España). Journal of Bat Research \& Conservation, 11(1): 10-13. https://doi.org/10.14709/BarbJ.11.1.2018.02

GEBHARD, J. \& BOGDANOWICZ, W. (2004). Nyctalus noctula (Schreber, 1774) Großer Abendsegler. In: Handbuch der Säugetiere Europas. Fledertiere II. ed.: Niethammer, J. \& Krapp, F. Wiebelsheim, Germany, p.605-694.

CAMPRODON, J. \& GUIXÉ, D. (2007). Ecologia i conservació dels quiròpters forestals. Departament de Medi Ambient i Habitatge. Generalitat de Catalunya. Capítol I: La comunitat de ratpenats a les fagedes (2006 i 2007). Capítol II: La comunitat de ratpenats a les pinedes de pi negre. Inédito.

CAMPRODON, J. \& GUIXÉ, D. (2008). Ecologia i conservació dels quiròpters forestals. Departament de Medi Ambient i Habitatge. Generalitat de Catalunya. Control de refugis i captures en fagedes i pinedes de pi negre, ecolocalització i instal-lació de refugis en rouredes. Inédito.

GUIXÉ, D. \& CAMPRODON, J. (2018). Manual de conservación y seguimiento de los quirópteros forestales. ed.: Ministerio de Agricultura, Pesca y Alimentación; Ministerio para la Transición Ecológica. Madrid, España, $274 \mathrm{pp}$.

HORN, J. W., ARNETT, E. B. \& KUNZ, T. H. (2008). Behavioral responses of bats to operating wind turbines. Journal of Wildlife Management, 72(1): 123-132. https://doi. org/10.2193/2006-465

IBÁÑEZ, C., GUILLÉN, A., FERNÁNDEZ, R., PÉREZ, J. L. \& GUERRERO, S. I. (1992). Iberian distribution of some little known bat species. Mammalia, 56(3): 433-444. https://doi.org/10.1515/mamm.1992.56.3.433

IBÁÑEZ, C., JUSTE, J., GARCÍA-MUDARRA, J. L. \& AGIRREMENDI, P. T. (2001). Bat predation on nocturnally migrating birds. PNAS, 98(17): 9700-9702. https://doi. org/10.1073/pnas.171140598

IBÁÑEZ, C., GUILLÉN, A., AGIRRE-MENDI, P.T., JUSTE, J., SCHREUR, G., CORDERO, A.I., POPA-LISSEANU, A.P. (2009). Sexual Segregation in Iberian Noctule Bats, Journal of Mammalogy, 90 (1): 235-243. https:// doi.org/10.1644/08-MAMM-A-037.1

JOHNSON, J. B., FORD, W. M., RODRIGUES, J. L. \& EDWARDS, J. W. (2012). Effects of acoustic deterrents on foraging bats. ed.: Department of Agriculture, Forest Service, Northern Research Station. Newtown Square, Pennsylvania, United States, 5 pp. https://doi. org/10.2737/NRS-RN-129

JUNTA DE CASTILLA Y LEÓN (2019). Base de datos de los planes de seguimiento de los parques eólicos de la provincia de Soria. Consejería de Fomento y Medio Ambiente, Delegación Territorial de Medio Ambiente de Soria.
JUSTE, J. (2007). Nyctalus lasiopterus (Schreber, 1780). En: Atlas y Libro Rojo de los Mamíferos Terrestres de España. ed.: Dirección General para la Biodiversidad-SECEMSECEMU. Madrid, España, p.233-236.

KRONWITTER, F. (1988). Population structure, habitat use and activity patterns of the noctule bat, Nyctalus noctula, revealed by radio-tracking. Myotis, 26: 23-85.

LORENTE, L., LLANA, C. \& SÁNCHEZ, J. M. (2014). Primera cita de nóctulo mediano (Nyctalus noctula) en Aragón (NE de España). Journal of Bat Research \& Conservation, 7(1): 19-21. https://doi.org/10.14709/BarbJ.7.1.2014.04

MEIJIDE, M., MEIJIDE, F., CLAVEL, F. \& GARCÍA-ASENSIO, J. M. (1996). Atlas preliminar de los mamíferos de Soria (España). Doñana, Acta Vertebrata, 23(2): 253-282.

MOLLEDA, R. \& FOMBELLIDA, I. (2018). Contribución al conocimiento de la distribución y estatus de la fauna quiropterológica de la Comunidad Autónoma de Cantabria. Journal of Bat Research \& Conservation, 11(1): 19-38. https://doi.org/10.14709/BarbJ.11.1.2018.04

NICHOLLS, B. \& RACEY. P. A. (2007). Bats avoid radar installations: could electromagnetic fields deter bats from colliding with wind turbines? PLOS ONE, 2(3): e297. https://doi.org/10.1371/journal.pone.0000297

NICHOLLS, B. \& RACEY, P. A. (2009). The aversive effect of electromagnetic radiation on foraging bats - $A$ possible means of discouraging bats from approaching wind turbines. PLOS ONE, 4(7): e6246. https://doi. org/10.1371/journal.pone.0006246

PALOMO, L. J., GISBERT, J. \& BLANCO, J. C. (2007). Atlas y Libro Rojo de los Mamíferos Terrestres de España. ed.: Dirección General para la Biodiversidad-SECEM-SECEMU. Madrid, España, 588 pp.

POPA-LISSEANU, A.G., DELGADO-HUERTAS, A-, FORERO, M.G., RODRÍGUEZ, A., ARLETTAZ, R., IBÁÑEZ, C. (2007). Bats' conquest of a formidable foraging niche: The myriads of nocturnally migrating songbirds. PLOS ONE 2(2): e205. https://doi.org/10.1371/journal. pone.0000205

POPA-LISSEANU, A. G., BONTADINA, F. \& IBÁÑEZ, C. (2009). Giant noctule bats face conflicting constraints between roosting and foraging in a fragmented and heterogeneous landscape. Journal of Zoology, 278: 126-133. https://doi. org/10.1111/j.1469-7998.2009.00556.x

RACHWALD, A. (1992). Habitat preference and activity of the noctule bat Nyctalus noctula in the Białowieża Primeval Forest. Acta Theriologica, 37(4): 413-422. https://doi. org/10.4098/AT.arch.92-42

RIVAS-MARTíNEZ, S. (1982). Étages bioclimatiques, secteurs chorologiques et séries de végetation de I'Espagne mediterranéenne. Ecología Mediterránea, 8(1-2): 275288. 
RODRIGUES, L., BACH, L., DUBOURG-SAVAGE, M., KARAPANDŽA, B., KOVAČ, D., KERVYN, T., DEKKER, J., KEPEL, A., BACH, P., COLLINS, J., et. al. (2015). Guidelines for consideration of bats in wind farm projects - Revisión 2014. Publication series No. 6. ed.: EUROBATS-UNEP. Bonn, Germany, $133 \mathrm{pp}$.

RUEDI, M., TUPINIER, Y. \& DE PAZ, O. (1998). First breeding record for the noctule bat (Nyctalus noctula) in the Iberian Peninsula. Mammalia, 62: 301-304.

SANTOS, J. D., MEYER, C. F. J., IBÁÑEZ, C., POPA-LISSEANU, A. G. \& JUSTE, J. (2016). Dispersal and group formation dynamics in a rare and endangered temperate forest bat (Nyctalus lasiopterus, Chiroptera: Vespertilionidae). Ecology and Evolution, 6(22): 8193-8204. https://doi. org/10.1002/ece3.2330
SLUITER, J. W. \& VAN HEERDT, P. F. (1966). Seasonal habits of the noctule bat (Nyctalus noctula). Archives Néerlandaises de Zoologie, 16(4): 423-439. https://doi. org/10.1163/036551666X00011

STRELKOV, P. (2000). Seasonal distribution of migratory bat species (Chiroptera, Vespertilionidae) in Eastern Europe and adjacent territories: nursing area. Myotis, 37: 7-25.

SZEWCZAK, J. M. \& ARNETT, E. B. (2008). Field test results of a potential acoustic deterrent to reduce bat mortality from wind turbines. ed.: Bat Conservation International. $14 \mathrm{pp}$. 